\title{
Error Analysis of Ultra Short Term Wind Power Prediction Model
}

\author{
Xiaofan Zhu ${ }^{1, a}$, Xiaoming Zha ${ }^{1, b}$, and Liang Qin ${ }^{1, c}$ \\ ${ }^{1}$ School of Electrical Engineering, Wuhan University, Wuhan, China \\ azxf8509@163.com, ${ }^{b}$ xmzha@whu.edu.cn, ${ }^{c}$ qinliang@whu.edu.cn
}

\begin{abstract}
Keywords: Error distribution; Sectional exponential distribution; Parameter estimation
Abstract. In this paper, we use a piecewise exponential distribution model to predict the ultra short term wind power error and then estimate the parameters. The case we used is from Northern Ireland, we forecast the probability and precision of wind power on the basis of Normal distribution model, Laplace distribution model, Cauchy distribution model, Beta distribution model and the proposed piecewise exponential distribution model. The prediction error distribution model of the sub index wind power forecasting error can be used to mine the relative information of the actual error distribution, in addition, it's convenient to implement and easy to be used in calculus, it can be applied to describe the error distribution of the multiple time scale prediction, so it has more advantages in the error analysis.
\end{abstract}

\section{Introduction}

Wind power has many advantages ${ }^{[1-2]}$; we all know that it's clean, economical and renewable, and the development scale of wind power is constantly expanding, however, wind power also has some disadvantages, such as the characteristic of its randomness, intermittency, non-scheduling and partial predictability, as a result, it brings severe challenges to the large-scale wind power gird for electricity dispatching operation. Compared with our country, many foreign countries carried out the research of wind power prediction error distribution earlier, the normal distribution, beta distribution, Cauchy distribution and Laplace distribution and several traditional empirical distribution models were proposed ${ }^{[3-6]}$. At the same time, different error distribution models have their own characteristics and the best applicable scope, the normal distribution assumption is the most widely used at present and it was put forward earliest. As to large scale wind farms, when the time scale is longer, the central limit theorem is affected, and the prediction error of the wind power will tend to be the normal distribution. What's more, the kurtosis of the normal distribution is fixed, with the in-depth study and a large number of statistics analysis, it is found that although the wind speed forecasting error close to the Gaussian distribution, but because of the power curve exhibits nonlinear behavior, it leads to the wind power prediction error do not follow Gaussian distribution, and it has some skewness as well, also, the confidence intervals on the prediction points is not symmetrical. The assumption of the normal distribution based on the prediction error of wind power, it will lead to the differential term of the objective function of the optimization problem be obtained by solving the differential term.

Of course, the error distribution may be a little bit fuzzy or have some skewness due to the different size of the wind farm and the prediction method, the choice of the error model should be based on the actual situation to select the most accurate description of the wind power error distribution model ${ }^{[7-9]}$. In this paper, a new adaptive and suitable error distribution model is proposed. It is hoped that the model has a more flexible shape, which can be used to describe the asymmetric error distribution, at the same time, at the same time; the form of the distribution should be simple, so as to easy to apply to engineering application. 


\section{Error analysis of ultra short term wind power prediction model}

In the error distribution model of the several traditional wind power prediction, the Normal distribution, Cauchy distribution and Laplace distribution is symmetrical distribution, Berta distribution error model can handle different skewness and kurtosis, which is a kind of weighted composition distribution, and it is also a hot spot in the research of wind power error. Traditional error distribution function model please refer to Appendix A, and the 4 conventional error models are shown in figure 1.

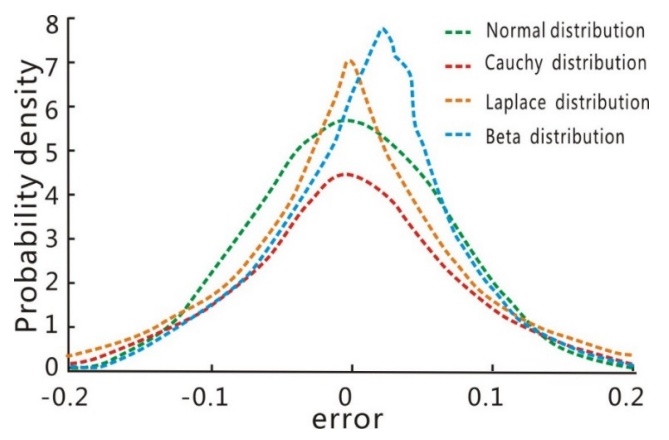

Fig.1 Four kinds of error distribution models for wind power forecasts

The symmetric error distribution model describes the error distribution of the research object, in the premise of ensuring certain accuracy; its scope of application is limited. Because most of the wind farm is not too large, the error distribution may have many different distribution characteristics. When the error distribution is asymmetric, even using the best parameter estimation method, the normal distribution, Cauchy distribution and Laplace distribution will still have a large deviation, which cannot more accurately describe the actual error distribution. In this paper, the model of the distribution of the sub exponential distribution and the Berta distribution can handle the asymmetric distribution. But the latter is complex and in the process of synthesis, the probability density is sometimes abnormal, at the same time, in the process of data processing, the error information of the wind power prediction is ignored, so as to ensure the final error distribution. As a result of the lack of information, the description accuracy will affect the beta error distribution model in a certain extent.

We hope to present a more flexible shape, convenient solution, variable kurtosis and wide application range distribution model; it requires that the new model can excavate the information about the actual error distribution, meanwhile, the shape of the error model can be modified with the change of the shape of the actual error distribution, hence, it can be used to describe the error distribution of wind power in most of the research object. The exponential error distribution model is the synthesis of the two segment exponential distribution and it has two shape parameters, what's more, the shape parameter of the two section index distribution is estimated, so the sub exponential distribution is more flexible than the traditional model.

The probability density function of the exponential distribution is assumed:

$$
f(x)=\left\{\begin{array}{cc}
a_{1} \exp \left(\frac{x-\mu_{0}}{b_{1}}\right) & x<\mu_{0} \\
a_{2} \exp \left(-\frac{x-\mu_{0}}{b_{2}}\right) & x \geq \mu_{0}
\end{array}\right.
$$

The formula (1) shall be satisfied: 1 .the integral of the negative infinity is $1 ; 2$.in the two section of the exponential distribution of the joint point, the probability density function of the two sections is equal to the value of the function. Therefore, the probability density functions of piecewise exponential distribution is: 


$$
f(x)=\left\{\begin{array}{cc}
\frac{1}{b_{1}+b_{2}} \exp \left(\frac{x-\mu_{0}}{b_{1}}\right) & x<\mu_{0} \\
\frac{1}{b_{1}+b_{2}} \exp \left(-\frac{x-\mu_{0}}{b_{2}}\right) & x \geq \mu_{0}
\end{array}\right.
$$

In this formula, $x=\left(P_{\text {forecast }}-P_{\text {actual }}\right) / P_{\text {cap }}$ is a standardized wind power prediction error, of which, $P_{\text {forecast }}$ is the wind power forecasting of wind farm, $P_{\text {actual }}$ is the measured value of wind power, $P_{\text {cap }}$ is wind power installed capacity, $b_{1}$ and $b_{2}$ for the evaluating of the shape parameter, which determines the shape of the curve; $\mu_{0}$ is the combination of the two segment exponential distribution, that is, when the relative error is $\mu_{0}$, the two stage exponential distribution joined, $\mu_{0}$ takes the maximum probability density point of the actual probability density sequence as the corresponding standard error value.

When $b_{1=} b_{2}=b$, this distribution is Laplace distribution, so Laplace distribution is a special case of the exponential distribution. Based on the probability density function (formula (2)), the cumulative probability distribution function of the exponential distribution model is derived:

$$
F(x)=\int_{-\infty}^{x} f(x) d x=\left\{\begin{array}{cl}
\frac{b_{1}}{b_{1}+b_{2}} \exp \left(\frac{x-\mu_{0}}{b_{1}}\right) & x<\mu_{0} \\
1-\frac{b_{2}}{b_{1}+b_{2}} \exp \left(-\frac{x-\mu_{0}}{b_{2}}\right) & x \geq \mu_{0}
\end{array}\right.
$$

The error distribution model of the wind power forecast of the sub index is composed of two sections, and the characteristics of this model are: 1.the partial parameters of the two stage exponential distribution are estimated by the two sets of independent error sequences, so the two sections is independent of the shape. 2. Taking advantage of the "watershed" which exists in the actual error distribution---the maximum peak value of probability density distribution, then determine the binding point of the two segments, based on this characteristic, compared with the traditional model, the new model has its own advantages, such as its Kurtosis is variable, the shape is flexible and it is easy to solve the outcomes.

\section{Case analysis}

This case was taken from the wind power forecast and actual measurement data from the (EirGrid) wind farm in 2010, 2011, 2012 (January to August $19^{\text {th }}$ ), the research object is to analyze the measured data and forecast data from the same period of time.

According to the probability density sequence of $X$ corresponding to the actual error sequence $Y$, the actual error probability density can be obtained as shown in Figure 2:

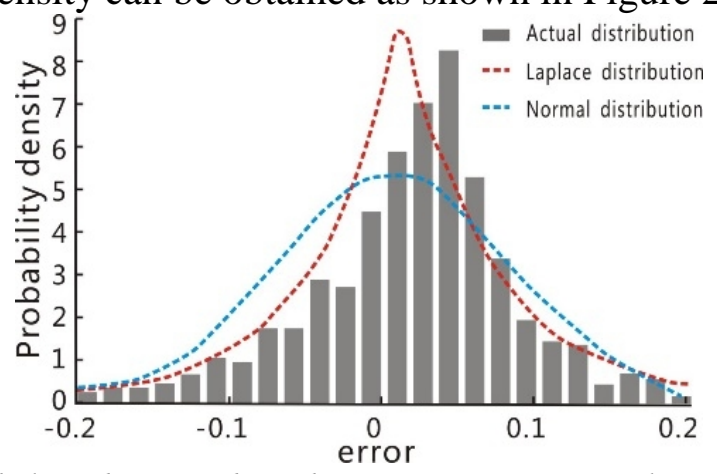

Fig.2 Comparison of probability density distribution among actual error distribution, normal and

\section{Laplace model}

It can be seen from figure 2 that the actual error distribution characteristics are as follows: 1 . the kurtosis of the normal distribution is 3 and the kurtosis of the Laplace distribution is 6 , so the kurtosis of the distribution is between 3-6; 2. This distribution is asymmetric and has a certain bias, meanwhile, 
the fitting accuracy can be reduced by using the symmetric error distribution model. Therefore, the error distribution is represented by the normal distribution and Laplace distribution, and the deviation of the actual distribution of the errors is larger.

From figure 2 and figure 3, the results show that for the case of wind power prediction error distribution's situation. The longitudinal and transverse error of probability density distribution of sub exponential probability, which is relatively small compared with the normal distribution, Laplace distribution, Cauchy distribution and beta distribution. This point can be seen from figure 2 and figure 3 , which shows that the accuracy of the probability density distribution of the sub index is higher than the other models. The reason is that the exponential coefficient of the two section index distribution is different and the shape is more flexible.

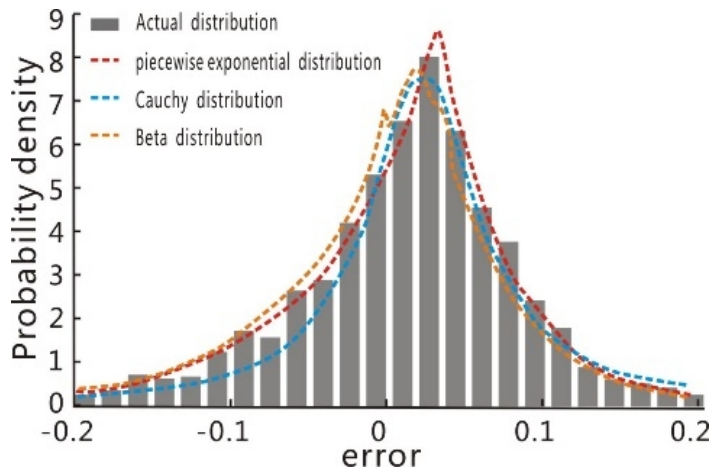

Fig.3 Comparison of probability density distribution among actual error distribution, piecewise exponential and Cauchy model

Compared table 3 and figure 4, table 3 shows that as to the wind power prediction error distribution, the longitudinal and transverse error of the cumulative probability distribution of the exponential distribution is obviously smaller than the normal distribution and Cauchy distribution. From Figure 4 it can be seen that the cumulative probability distribution of the exponential distribution and the actual error distribution are basically coincident, meanwhile, there is a larger deviation between the Normal distribution, Cauchy distribution and the actual cumulative probability distribution.

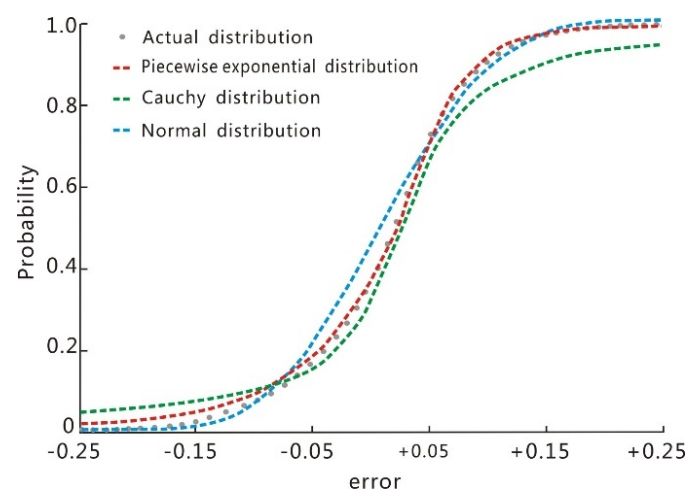

Fig.4 Comparison of cumulative probability distribution among actual error distribution, piecewise exponential, Cauchy and normal model

Compared table 3 and figure 5, the result of table 3 show that as to the wind power prediction error distribution in this case, the longitudinal and transverse error of the cumulative probability distribution of the exponential distribution is very similar to t Laplace distribution, and both are very similar, at the same time, they are both smaller than Beta distribution, which can be seen from figure 5. 


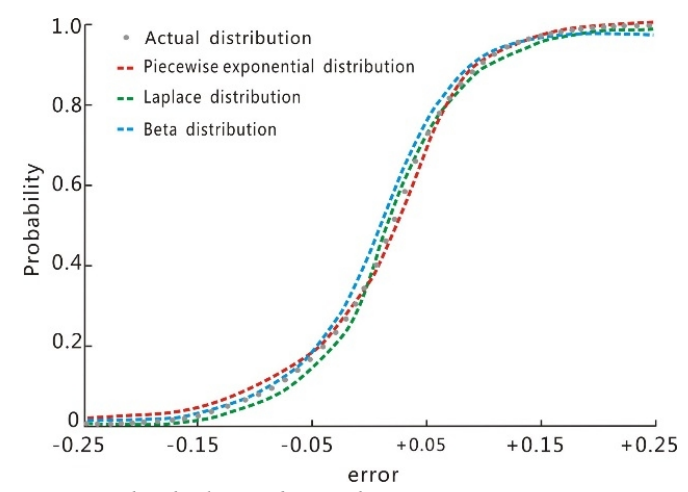

Fig.5 Comparison of cumulative probability distribution among actual error distribution, piecewise exponential, Laplace and Beta model

From the above analysis we can make the conclusion that the fitting accuracy of the exponential cumulative probability distribution and the cumulative probability distribution of Laplace is much higher than the normal distribution and Cauchy distribution in this case, and it slightly higher than the beta distribution, but the fitting precision of Laplace probability density distribution is relatively low. Therefore, in this case, Laplace distribution model cannot guarantee the accuracy of probability density distribution and cumulative probability distribution, the probability density distribution and cumulative probability distribution of Berta distribution can meet the requirements of the accuracy of the distribution, but compared with the piecewise exponential distribution, the accuracy should be slightly inferior and the solving process is relatively complex. The piecewise exponential distribution is estimated by the shape parameter estimation, so the figure is flexible, and at the same time, it can also guarantee the precision of the probability density distribution and the probability distribution.

\section{Conclusions}

Influenced by the scale of wind farm, the scale of the forecasting time and the degree of abandoned wind, the prediction error distribution of different wind farms showed different characteristics; and the traditional error model cannot meet the requirements of the vast majority of asymmetric error distribution. In this paper, we propose a model for the prediction of the error distribution of the sub exponential wind power. This model can be used to mine the actual error distribution, in addition, the shape is flexible and there is no fixed demand to the error distribution, skewness and kurtosis characteristics, the solution is convenient, which is easy to be used in calculus, and it is suitable for engineering application. These features make the model can be used to describe the error distribution of the multiple prediction time scale, and it has more advantages in describing asymmetric error distribution.

\section{Acknowledgements}

This work was financially supplied by Scientific Funds for Outstanding Young Scientists of China (51207115).

\section{References}

[1] Zhang H, Chen L, Qu Y, et al. Support Vector Regression Based on Grid-Search Method for Short-Term Wind Power Forecasting[J]. Journal of Applied Mathematics, 2014, 2014.

[2] Ren C, An N, Wang J, et al. Optimal parameters selection for BP neural network based on particle swarm optimization: A case study of wind speed forecasting[J]. Knowledge-Based Systems, 2014, 56: 226-239. 
[3] Liu J, Meng H, Hu Y, et al. A novel MPPT method for enhancing energy conversion efficiency taking power smoothing into account[J]. Energy Conversion and Management, 2015, 101: 738-748.

[4] Pathak A K, Sharma M P, Bundele M. A critical review of voltage and reactive power management of wind farms[J]. Renewable and Sustainable Energy Reviews, 2015, 51: 460-471.

[5] Wang J Z, Wang Y, Jiang P. The study and application of a novel hybrid forecasting model-A case study of wind speed forecasting in China[J]. Applied Energy, 2015, 143: 472-488.

[6] Zhao B, Shi Y, Dong X, et al. Short-term operation scheduling in renewable-powered microgrids: a duality-based approach[J]. Sustainable Energy, IEEE Transactions on, 2014, 5(1): 209-217.

[7] Ata R. Artificial neural networks applications in wind energy systems: a review[J]. Renewable and Sustainable Energy Reviews, 2015, 49: 534-562.

[8] Marjanovic N, Wharton S, Chow F K. Investigation of model parameters for high-resolution wind energy forecasting: Case studies over simple and complex terrain[J]. Journal of Wind Engineering and Industrial Aerodynamics, 2014, 134: 10-24.

[9] Fan X, Wang W, Shi R, et al. Review of developments and insights into an index system of wind power utilization level[J]. Renewable and Sustainable Energy Reviews, 2015, 48: 463-471. 